\title{
Advances in anti-Ramsey theory for random graphs
}

\author{
Guilherme Oliveira Mota ${ }^{1,2}$ \\ ${ }^{1}$ Centro de Matemática, Computação e Cognição, Universidade Federal do ABC \\ Santo André, Brazil \\ ${ }^{2}$ Instituto de Matemática e Estatística, Universidade de São Paulo \\ São Paulo, Brazil \\ mota@ime.usp.br
}

\begin{abstract}
Given graphs $G$ and $H$, we denote the following property by $G \underset{\mathrm{p}}{\mathrm{rb}} H$ : for every proper edge-colouring of $G$ (with an arbitrary number of colours) there is a rainbow copy of $H$ in $G$, i.e., a copy of $H$ with no two edges of the same colour. It is known that, for every graph $H$, the threshold function $p_{H}^{\mathrm{rb}}=$ $p_{H}^{\mathrm{rb}}(n)$ of this property for the binomial random graph $G(n, p)$ is asymptotically at most $n^{-1 / m^{(2)}(H)}$, where $m^{(2)}(H)$ denotes the so-called maximum 2-density of $H$. In this work we discuss this and some recent results in the study of antiRamsey properties in random graphs, and we prove that if $H=C_{4}$ or $H=K_{4}$ then $p_{H}^{\mathrm{rb}}<n^{-1 / m^{(2)}(H)}$, which is in contrast with the known facts that $p_{C_{k}}^{\mathrm{rb}}=$ $n^{-1 / m^{(2)}\left(C_{k}\right)}$ for $k \geqslant 7$, and $p_{K_{\ell}}^{\mathrm{rb}}=n^{-1 / m^{(2)}\left(K_{\ell}\right)}$ for $k \geqslant 19$.
\end{abstract}

Resumo. Dados grafos $G$ e $H$, denotamos a seguinte propriedade por $G \underset{\mathrm{p}}{\mathrm{rb}} H$ : para toda coloração própria das arestas de $G$ (com uma quantidade arbitrária de cores) existe uma cópia multicolorida de $H$ em $G$, i.e., uma cópia de $H$ sem duas arestas da mesma cor. Sabe-se que, para todo grafo $H$, a função limiar $p_{H}^{\mathrm{rb}}=p_{H}^{\mathrm{rb}}(n)$ para essa propriedade no grafo aleatório binomial $G(n, p)$ é assintoticamente no máximo $n^{-1 / m^{(2)}(H)}$, onde $m^{(2)}(H)$ denota a assim chamada 2-densidade máxima de $H$. Neste trabalho discutimos esse e alguns resultados recentes no estudo de propriedades anti-Ramsey para grafos aleatórios, e mostramos que se $H=C_{4}$ ou $H=K_{4}$ então $p_{H}^{\mathrm{rb}}<n^{-1 / m^{(2)}(H)}$, que está em contraste com os fatos conhecidos de que $p_{C_{k}}^{\mathrm{rb}}=n^{-1 / m^{(2)}\left(C_{k}\right)}$ para $k \geqslant 7$, e $p_{K_{\ell}}^{\mathrm{rb}}=n^{-1 / m^{(2)}\left(K_{\ell}\right)}$ para $k \geqslant 19$.

\section{Introduction}

Let $r$ be a positive integer and let $G$ and $H$ be graphs. We denote by $G \rightarrow(H)_{r}$ the property that any colouring of the edges of $G$ with at most $r$ colours contains a monochromatic copy of $H$ in $G$. In 1995, Rödl and Ruciński determined the threshold for the property $G(n, p) \rightarrow(H)_{r}$ for all graphs $H$. The maximum 2-density $m^{(2)}(H)$ of a graph $H$ is denoted by $m^{(2)}(H)=\max \left\{\frac{|E(J)|-1}{|V(J)|-2}: J \subset H,|V(J)| \geqslant 3\right\}$, where we suppose $|V(H)| \geqslant 3$.

The author was supported by FAPESP (2013/11431-2, 2013/03447-6) and partially by CNPq (459335/2014-6). The author acknowledges the support of CAPES/DAAD PROBRAL (430/15). 
Theorem 1 (Rödl and Ruciński [Rödl and Ruciński 1993, Rödl and Ruciński 1995]). Let $H$ be a graph containing a cycle. Then, the threshold function $p_{H}=p_{H}(n)$ for the property $G(n, p) \rightarrow(H)_{r}$ is given by $p_{H}(n)=n^{-1 / m^{(2)}(H)}$.

Given a graph $H$, we are interested in the following 'anti-Ramsey' type properties of the random graph $G=G(n, p)$, denoted by $G \underset{\mathrm{p}}{\stackrel{\mathrm{rb}}{\mathrm{b}}} H$ : for every proper edgecolouring of $G$, there exists a rainbow copy of $H$ in $G$, i.e., a copy of $H$ with no two edges of the same colour. The term 'anti-Ramsey' is used in different contexts, but we follow the terminology used in [Kohayakawa et al. 2014, Kohayakawa et al. 2017, Nenadov et al. 2017, Rödl and Tuza 1992]. Since the property $G(n, p) \underset{\mathrm{p}}{\mathrm{rb}} H$ is increasing for every fixed graph $H$, we know that it admits a threshold function $p_{H}^{\mathrm{rb}}=$ $p_{H}^{\mathrm{rb}}(n)$ [Bollobás and Thomason 1987].

The study of anti-Ramsey properties of random graphs was initiated by Rödl and Tuza, who proved in [Rödl and Tuza 1992] that for every $\ell$ there exists a fairly small $p$, such that $G(n, p) \underset{\mathrm{p}}{\mathrm{rb}} C_{\ell}$ almost surely. In fact, this result answers positively a question posed by Spencer (see [Erdôs 1979], p. 29), who asked whether there are graphs of arbitrarily large girth that contain a rainbow cycle in any proper edge-colouring. We obtained the following result, which implies that $p_{H}^{\mathrm{rb}} \leqslant n^{-1 / m^{(2)}(H)}$ for any fixed graph $H$.

Theorem 2 (Kohayakawa, Konstadinidis and Mota [Kohayakawa et al. 2014]). If $H$ is a fixed graph, then there exists a constant $C>0$ such that for $p=p(n) \geqslant C n^{-1 / m^{(2)}(H)}$ we asymptotically almost surely have $G(n, p) \underset{\mathrm{p}}{\mathrm{rb}} H$.

The proof of Theorem 2 combines ideas from the regularity method for sparse graphs (see, e.g., [Kohayakawa 1997, Kohayakawa and Rödl 2003, Szemerédi 1978]) and a characterization of quasi-random sparse graphs (see, e.g., [Chung and Graham 2008]). This result was the beginning of a systematic study about anti-Ramsey problems in random graphs. In [Kohayakawa et al. 2017] we proved that for an infinite family of graphs $F$ we have $p_{F}^{\text {rb }} \ll n^{-1 / m^{(2)}(F)}$, which is in contrast with Theorem 1. Before state this result precisely we need one more definition: given a graph $H$ with $m^{(2)}(H)<2$, put $\beta\left(H, K_{3}\right)=\frac{1}{3}\left(1+\frac{1}{m^{(2)}(H)}\right)$. Theorem 3 below makes the discussion above precise.

Theorem 3. Suppose $k \geqslant 4$ and let $F$ be the $(k+1)$-vertex graph composed by a $k$ vertex graph $H$ with $1<m^{(2)}(H)<2$ and a vertex outside of $H$ that is adjacent to two adjacent vertices of $H$. Then, for a suitably large constant $D$, if $p \geqslant D n^{-\beta\left(H, K_{3}\right)}$, then $G(n, p) \underset{\mathrm{p}}{\mathrm{rb}}$ F almost surely.

We can easily conclude that for graphs $F$ as in the statement of Theorem 3 we have $p_{F}^{\mathrm{rb}} \ll n^{-1 / m^{(2)}(F)}$ since one can check that $1 / m^{(2)}(F)=1 / m^{(2)}\left(K_{3}\right)=1 / 2<$ $\beta\left(H, K_{3}\right)<1 / m^{(2)}(H)$. This makes the following question interesting: What are the graphs $H$ for which $p_{H}^{\mathrm{rb}}=n^{-1 / m^{(2)}(H)}$ ? Recently, some progress in answering this question was made in [Nenadov et al. 2017], which proved the following result.

Theorem 4 (Nenadov, Person, Škorić and Steger [Nenadov et al. 2017]). Let H be a cycle on at least 7 vertices or a complete graph on at least 19 vertices. Then $p_{H}^{\mathrm{rb}}=n^{-1 / m^{(2)}(H)}$.

The authors of Theorem 4 remarked that their result could hold for all cycles and cliques of size at least 4 . We conjecture that Theorem 4 can indeed be extended to cycles 
and cliques of size at least 5 , but not for $C_{4}$ and $K_{4}$. In fact, we show that if $H$ is $C_{4}$ or $K_{4}$, then $p_{H}^{\mathrm{rb}}$ is asymptotically smaller than $n^{-1 / m^{(2)}(H)}$.

Theorem 5. We have $p_{C_{4}}^{\mathrm{rb}}=n^{-3 / 4}$ and $p_{K_{4}}^{\mathrm{rb}}=n^{-7 / 15}$.

In what follows we give a brief outline of the proof of Theorem 5 for cycles $C_{4}$. We remark that the proof for $K_{4}$ makes use of similar techniques.

\section{Brief outline of the proof of Theorem 5 for $C_{4}$}

First, we consider the density $m(H)$ of a graph $H$, defined as $m(H)=$ $\max \left\{\frac{|E(J)|}{|V(J)|}: J \subset H,|V(J)| \geqslant 1\right\}$. We will use of the following result.

Theorem 6 (Bollobás [Bollobás 2001]). Let $H$ be a fixed graph. Then, $p=n^{-1 / m(H)}$ is the threshold for the property that $G$ contains a copy of $H$.

Note that for proving the upper bounds it is enough to show that $G(n, p)$ a.s. contains a small graph that forces a rainbow copy of the given graphs in any proper edgecolouring. Since the proof for the upper bounds are much simpler than the proof for the lower bounds, we give the full proof of the upper bound in the case of $C_{4}$.

\section{Upper bound for $p_{C_{4}}^{\mathrm{rb}}$.}

Consider the complete bipartite graph $K_{2,4}$ with partition classes $\{a, b\}$ and $\{w, x, y, z\}$. We will first show that any proper colouring of the edges of $K_{2,4}$ contains a rainbow copy of $C_{4}$ and then we conclude that for $p \gg n^{-3 / 4}$ a.s. $G(n, p)$ contains a copy of $K_{2,4}$. Suppose by contradiction that there is a proper colouring $\chi$ of $E\left(K_{2,4}\right)$ with no rainbow copy of $C_{4}$. W.l.o.g. let $\chi(a w)=\chi(b x)=1$ and $\chi(a y)=\chi(b z)=2$. Since the colouring is proper the edges $a x$ and $a z$ get different new colours, say, $\chi(a x)=3$ and $\chi(a z)=4$. Since the $C_{4}$ induced by $\{a, x, b, y\}$ is not rainbow, we have $\chi(b y)=3$. But then the $C_{4}$ induced by the vertices $\{a, x, b, z\}$ is rainbow, a contradiction. Therefore, any colouring of the edges of $K_{2,4}$ contains a rainbow $C_{4}$. By Theorem 6 , if $p \gg n^{-3 / 4}$, then a.s. $G(n, p)$ contains a copy of $K_{2,4}$. Therefore, a.s. any proper colouring of the edges of $G(n, p)$ contains a rainbow copy of $C_{4}$, which implies that $p_{C_{4}}^{\mathrm{rb}} \leqslant n^{-1 / m\left(K_{2,4}\right)}=n^{-3 / 4}$.

\section{Lower bound for $p_{C_{4}}^{\mathrm{rb}}$.}

Now let us turn our attention to the lower bounds. Let $G$ and $H$ be graphs. We say that a sequence $F=H_{1}, \ldots, H_{\ell}$ of $H$-copies in $G$ is an $H$-chain if for any $2 \leqslant i \leqslant \ell$ we have $E\left(H_{i}\right) \cap\left(E\left(H_{1}\right), \ldots, E\left(H_{i-1}\right)\right) \neq \varnothing$. Note that a copy of $H$ in $G$ that does not intersect edge-wise with any other copy of $H$ is a maximal $H$-chain composed by only one copy of $H$. Furthermore, the edge sets of two distinct maximal $H$-chains are disjoint. Thus, it is easy to see that each $H$ in $G$ belongs to exactly one maximal $H$-chain.

Let $G=G(n, p)$ and let $p \ll n^{-3 / 4}$. The idea is to prove that a.s. there exists a proper colouring of $G$ that contains no rainbow $C_{4}$. In this proof we will consider $C_{4^{-}}$ chains that are maximal with respect to the number of $C_{4}$ 's. The first and more important step is to colour some edges in all maximal $C_{4}$-chains so that all $C_{4}$ 's in $G$ will be nonrainbow and this partial colouring will be proper. Then, since all $C_{4}$ 's are coloured we can just give a new colour for each one of the remaining uncoloured edges. For the first step, we use Markov's inequality and the union bound to obtain that a.s.

$G$ does not contains any graph $H$ with $m(H) \geqslant 4 / 3$ and $|V(H)| \leqslant 12$. 
Let $F=C_{4}^{1}, \ldots, C_{4}^{\ell}$ be an arbitrary $C_{4}$-chain in $G$ with $m(F) \geqslant 4 / 3$. Let $2 \leqslant i \leqslant \ell$ be the smallest index such that $F^{\prime}=C_{4}^{1}, \ldots, C_{4}^{i}$ has density $m\left(F^{\prime}\right) \geqslant 4 / 3$. Then, since $F^{\prime \prime}=C_{4}^{1}, \ldots, C_{4}^{i-1}$ has density $m\left(F^{\prime \prime}\right)<4 / 3$, we can explore the structure of $G(n, p)$ to conclude that $\left|V\left(F^{\prime \prime}\right)\right| \leqslant 10$, which implies $\left|V\left(F^{\prime}\right)\right| \leqslant 12$, a contradiction with (1). Therefore, a.s. $G(n, p)$ contains no copy of $C_{4}$-chains $F$ with $m(F) \geqslant 4 / 3$. Thus, we may assume that all $C_{4}$-chains $F$ of $G$ have density $m(F)<4 / 3$. In this case, it is possible to analyze carefully the structure of such chains, obtaining the desired colouring, which proves the claimed result.

\section{References}

Bollobás, B. (2001). Random graphs. Cambridge Studies in Advanced Mathematics. Cambridge University Press.

Bollobás, B. and Thomason, A. (1987). Threshold functions. Combinatorica, 7(1):35-38.

Chung, F. and Graham, R. (2008). Quasi-random graphs with given degree sequences. Random Structures \& Algorithms, 32(1):1-19.

Erdős, P. (1979). Some old and new problems in various branches of combinatorics. In Proc. 10th southeastern conference on combinatorics, graph theory and computing, pages 19-37, Winnipeg, Man. Utilitas Math.

Kohayakawa, Y. (1997). Szemerédi's regularity lemma for sparse graphs. In Foundations of computational mathematics (Rio de Janeiro, 1997), pages 216-230. Springer, Berlin.

Kohayakawa, Y., Konstadinidis, P. B., and Mota, G. O. (2014). On an anti-Ramsey threshold for random graphs. European Journal of Combinatorics, 40:26-41.

Kohayakawa, Y., Konstadinidis, P. B., and Mota, G. O. (2017). On an anti-ramsey threshold for sparse graphs with one triangle. Journal of Graph Theory. In press.

Kohayakawa, Y. and Rödl, V. (2003). Szemerédi's regularity lemma and quasirandomness. In Recent advances in algorithms and combinatorics, volume 11 of CMS Books Math./Ouvrages Math. SMC, pages 289-351. Springer, New York.

Nenadov, R., Person, Y., Škorić, N., and Steger, A. (2017). An algorithmic framework for obtaining lower bounds for random Ramsey problems. J. Combin. Theory Ser. B, 124:1-38.

Rödl, V. and Ruciński, A. (1993). Lower bounds on probability thresholds for Ramsey properties. In Combinatorics, Paul Erdös is eighty, Vol. 1, Bolyai Soc. Math. Stud., pages 317-346. János Bolyai Math. Soc., Budapest.

Rödl, V. and Ruciński, A. (1995). Threshold functions for Ramsey properties. J. Amer. Math. Soc., 8(4):917-942.

Rödl, V. and Tuza, Z. (1992). Rainbow subgraphs in properly edge-colored graphs. Random Structures Algorithms, 3(2):175-182.

Szemerédi, E. (1978). Regular partitions of graphs. In Problèmes combinatoires et théorie des graphes (Colloq. Internat. CNRS, Univ. Orsay, Orsay, 1976), volume 260 of Colloq. Internat. CNRS, pages 399-401. CNRS, Paris. 